\title{
Miranda
}

Revue pluridisciplinaire du monde anglophone /

Multidisciplinary peer-reviewed journal on the English-

speaking world

1 | 2010

Variations on Darwin

\section{Rae Beth Gordon, Dances with Darwin, 1875-1910: \\ Vernacular Modernity in France}

\section{Fanny Robles}

\section{OpenEdition}

Journals

Édition électronique

URL : http://journals.openedition.org/miranda/482

DOI : $10.4000 /$ miranda.482

ISSN : 2108-6559

Éditeur

Université Toulouse - Jean Jaurès

\section{Référence électronique}

Fanny Robles, «Rae Beth Gordon, Dances with Darwin, 1875-1910: Vernacular Modernity in France»,

Miranda [En ligne], 1 | 2010, mis en ligne le 23 mars 2010, consulté le 16 février 2021. URL : http:// journals.openedition.org/miranda/482 ; DOI : https://doi.org/10.4000/miranda.482

Ce document a été généré automatiquement le 16 février 2021.

\section{(c) (i) (3)}

Miranda is licensed under a Creative Commons Attribution-NonCommercial-NoDerivatives 4.0 International License. 


\title{
Rae Beth Gordon, Dances with Darwin, 1875-1910: Vernacular Modernity in France
}

\author{
Fanny Robles
}

\section{RÉFÉRENCE}

Rae Beth Gordon, Dances with Darwin, 1875-1910: Vernacular Modernity in France(Farnham:

Ashgate, 2009), 311 p, ISBN 978-0-7546-5243-4

1 C'est à l'occasion du bicentenaire de la naissance du célèbre scientifique victorien que Rae Beth Gordon publie Dances with Darwin, 1875-1910: Vernacular Modernity in France. L'auteur envisage son étude comme une véritable «anthropologie du geste » (" anthropology of gesture »), comme elle définit elle-même son entreprise dans sa préface. Dances with Darwin s'intéresse à l'esthétique du café-concert parisien de la fin $\mathrm{du} \mathrm{XIX}^{\mathrm{e}}$ siècle et du début $\mathrm{du} \mathrm{XX}^{\text {ème }}$, et fait converger culture populaire, histoire de la médecine et ethnologie, pour tenter de définir une esthétique évolutionniste du divertissement populaire français. L'ambition de Gordon, et ce qui fait toute l'originalité de sa démarche, est de resituer les débuts du modernisme dans ce contexte culturel particulier, un demi-siècle avant la naissance du surréalisme. Dances with Darwin est une étude abondamment illustrée, basée essentiellement sur des articles et illustrations provenant de journaux destinés à la classe moyenne, tels Le Courrier Français, Le Rire et Le Monde Illustré.

2 L'auteur se penche dans un premier temps sur la mode fin-de-siècle des chanteurs (et chanteuses) épileptiques - un genre musical né de la fascination pour les spectaculaires hystériques du Docteur Charcot. Les mouvements désarticulés des épileptiques sont souvent associés à la dégénérescence et à la sauvagerie, à la fois grotesque et sensuelle, des races inférieures. La maladie devient ainsi matière à théâtre, et même produit du théâtre, les spectateurs craignant (et recherchant, dans une 
certaine mesure) la contagion. La peur de l'infection, par l'imitation inconsciente du spectacle auquel on a été exposé, alimente les journaux de l'époque. Polaire, l'amie de Colette, devient le prototype de la femme fatale, hystérique et sauvage, qui jouit d'une immense gloire théâtrale.

Dans le chapitre suivant, Gordon se penche sur la théorie de l'évolution elle-même et son impact dans la société française de la fin du XIX ${ }^{e}$ siècle. L'auteur revient sur le débat relativement tardif autour de la question darwinienne, le scientifique britannique ayant longtemps été éclipsé en France par des prédécesseurs de renom comme Jean-Baptiste Lamarck. Davantage que l'Origine des Espèces, c'est la Filiation de l'Homme qui préoccupe les Français, et en particulier le lien que Charles Darwin instaure entre l'homme et le singe. Gordon s'intéresse alors aux "phénomènes » et autres "chaînons manquants" exhibés à l'époque en France et dans le reste de l'Europe. Souvent issus des empires britannique et français, ils offrent sur scène l'exemple d'un stade antérieur dans l'évolution de l'humanité, auquel l'homme moderne est susceptible de retourner s'il ne prend garde à la dégénérescence. Ce rôle de repoussoir est également tenu par les singes savants qui investissent la scène du music-hall.

Dans un troisième chapitre, Gordon aborde l'esthétique évolutionniste proprement dite, et son application au café-concert. Elle revient en particulier sur les idées de la psychophysique développées, à la suite de Gustav Theodor Fechner, par Charles Henry, James Sully et Grant Allen. Le principe fondamental de la psychophysique telle qu'elle est présentée par ces penseurs est l'origine purement physique du sens du beau: la beauté procure du plaisir alors que le laid provoque la douleur. Ainsi, les couleurs vives et le rythme musical saccadé des spectacles ethniques étaient associés au mauvais goût et à la souffrance du corps. Images d'une certaine dégénérescence, ils étaient aussi paradoxalement propices à la régénération des spectateurs neurasthéniques, par le stimulus visuel et (par conséquent) physique qu'ils leur infligeaient. Selon Gordon, les peintres Fauves illustrent parfaitement cette nouvelle esthétique.

5 L'auteur consacre les quatrième et cinquième chapitres à la présence des Noirs africains et américains dans la capitale française et aux liens qui associent le continent africain aux chanteurs épileptiques. Elle revient tout d'abord sur les villages ethniques des expositions universelles et coloniales, avant de s'attarder sur la célèbre figure du clown Chocolat. Elle en vient ensuite au succès parisien des minstrel shows dans les années 1870 et à la mode du cake-walk, une danse épileptique (l'élément de dislocation s'étant vu renforcé quand la danse a traversé l'Atlantique) venue des plantations américaines, qui passait pour être un divertissement de dégénérés. Gordon note que l'image de l'idéal féminin à la fin du XIX siècle était souvent celle d'un corps sauvage marqué par l'altérité raciale. Les cabarets parisiens étaient alors célèbres pour la quasi nudité de leurs chanteuses qui revendiquaient souvent leur origine étrangère, que ce soit l'Espagne pour la Belle Otéro ou l'Afrique du Nord pour Polaire - cette dernière incarnant l'hybridité qui, pour Gordon, caractérise déjà la fin du XIX ${ }^{\mathrm{e}}$ siècle.

Dans son dernier chapitre, l'auteur revient sur le personnage du Père Ubu et tente de déterminer dans quelle mesure les problématiques de la fin du XIX ${ }^{\mathrm{e}}$ siècle, qu'elle s'est efforcée d'étudier, se retrouvent dans le héros grotesque d'Alfred Jarry. La prégnance du corps et du laid se mêle chez le personnage à une fascination sans borne pour les théories scientifiques qui tournent chez lui à l'absurde. Tour à tour chaînon manquant et cannibale, suivant ses différentes incarnations, le Père Ubu semble transformer sa 
force de contagion corporelle et primaire en signe d'avant-garde et de modernité, régénérant l'art au tournant du siècle. Enfin, dans son épilogue, Gordon s'intéresse à la descendance artistique d'Ubu dans les mouvements futuriste, surréaliste et dadaïste, et dans le théâtre de la cruauté d'Antonin Artaud. La figure de Joséphine Baker vient clôturer l'étude, elle qui cristallise parfaitement de nombreux aspects de l'époque précédente.

7 En abordant la fin-de-siècle parisienne sous l'angle pluridisciplinaire des arts et des sciences, analysant l'influence des secondes sur les premiers, Rae Beth Gordon signe avec Dances with Darwin un ouvrage particulièrement original. La richesse des illustrations et la précision des références, aussi bien concernant les ouvrages scientifiques qu'artistiques, font de cette étude un outil de travail fort intéressant pour les chercheurs et étudiants, tant par les thèses innovantes qui y sont avancées que par l'originalité de la démarche qui n'hésite pas à multiplier les champs d'investigation.

\section{INDEX}

Keywords : Darwin, evolutionist aesthetics, café-concert, modernism, fin-de-siècle, epileptic song, hysteria, missing link, contagion, psychophysics, minstrel show, cake-walk, hybridity, avant-garde, Belle Otéro

Mots-clés : Darwin, esthétique évolutionniste, café-concert, modernisme, fin-de-siècle, chanson épileptique, hystérie, chaînon manquant, contagion, psychophysique, cake-walk, hybridité, avant-garde, Belle Otéro

\section{AUTEURS}

\section{FANNY ROBLES}

Allocataire-monitrice

Université Toulouse 2 -Le Mirail

fanny9robles@yahoo.fr 